\title{
CONVEX SOLUTIONS OF A SCHRÖDER EQUATION IN SEVERAL VARIABLES
}

\author{
FRED M. HOPPE 1
}

\begin{abstract}
A nonprobabilistic proof is given for the existence of the Yaglom conditional limit distribution for the subcritical multitype GaltonWatson process by using a uniqueness theorem for convex solutions of the multidimensional Schröder functional equation.
\end{abstract}

Introduction. An alternate proof is presented for the existence of the Yaglom conditional limit distribution in a subcritical positively regular, multitype Galton-Watson process. The original proof by Joffe and Spitzer (1967) required a probabilistic argument to obtain the convergence of a certain sequence of probability generating functions (p.g.f.'s). This was in contrast with the one-dimensional results of Heathcote, Seneta, and Vere-Jones (1967) who manifested this convergence as a consequence of convexity arguments pertaining to solutions of Schröder's functional equation (see Kuczma (1964)). The present proof is in the same analytic spirit although there is a marked difference in technique to account for the increased dimension. In particular, it is necessary to first establish the uniqueness of solutions to a Schröder equation and then to invoke this uniqueness to show that a sequence of difference quotients of iterates of p.g.f.'s converge. This paper is therefore another step in the program elegantly outlined by Seneta (1969) emphasizing functional equations as the appropriate setting for the study of many problems in branching processes. Other details in this direction may be found in the author's Princeton dissertation (1975b).

Background. $x \equiv\left(x_{1}, \ldots, x_{d}\right)$ will denote a generic point in $[0,1]^{d}$ with $0=(0, \ldots, 0)$ and $1=(1, \ldots, 1) . \quad x \leqslant y$ iff $x_{i} \leqslant y_{i}, 1 \leqslant i \leqslant d . \quad F(x)$ $\equiv\left(F^{(1)}(x), \ldots, F^{(d)}(x)\right)$ is the vector offspring p.g.f. and $F_{n}(x)$ denotes the $n$th functional iterate of $F(x)$. For notational convenience write $F_{n}(0)$ simply as $F_{n} . v$ and $u$ are the left and right eigenvectors, respectively, corresponding to the maximal eigenvalue $\rho<1$ of the offspring expectation matrix. Two basic asymptotic results of Joffe and Spitzer required below are:

Received by the editors October 27, 1976.

AMS (MOS) subject classifications (1970). Primary 60J20; Secondary 60F15.

Key words and phrases. Subcritical multitype Galton-Watson process, Yaglom limit, Schröder equation, convexity.

${ }^{1}$ Research supported in part by the Summer Research Institute of the Canadian Mathematical Congress at the Université de Montréal. 


$$
\lim _{n \rightarrow \infty}\left(1-F_{n}(x)\right) / v \cdot\left(1-F_{n}(x)\right)=u
$$

and

$$
\lim _{n \rightarrow \infty} v \cdot\left(1-F_{n+1}(x)\right) / v \cdot\left(1-F_{n}(x)\right)=\rho,
$$

both for $x \in[0,1]^{d}-1$.

It is required to show that for each $i$, the sequence

$$
\left\{\left(F_{n}^{(i)}(x)-F_{n}^{(i)}\right) /\left(1-F_{n}^{(i)}\right)\right\}_{n=1}^{\infty}
$$

tends to a limit which is a proper p.g.f. In one dimension, by convexity, if $0 \leqslant x \leqslant F(0)$, then

$$
\frac{1-F\left(F_{n}(x)\right)}{1-F_{n}(x)} \geqslant \frac{1-F\left(F_{n}\right)}{1-F_{n}}
$$

and cross-multiplication then shows that $(2 a)$ is a monotonic decreasing sequence, which establishes the limit. (Extension to all $x \in[0,1]$ is then straightforward in view of (1b).)

This argument fails in $\mathbf{R}^{d}$ because of a lack of monotonicity. To overcome this problem, Joffe and Spitzer allowed the index $i$ in (2a) to vary with $n$ in such a way as to obtain monotonicity. Their very clever selection of $i$ depended upon being able to express $F_{n}(x)$ as a p.g.f. and following up with an argument based on the Markov property.

The approach followed in this paper is as follows. By virtue of (1a) it suffices to work with the sequence

$$
\left\{v \cdot\left(F_{n}(x)-F_{n}\right) / v \cdot\left(1-F_{n}\right)\right\}_{n=0}^{\infty} .
$$

This sequence is also not monotonic. However the lim sup exists and can be identified as the unique convex and monotonic nondecreasing (with respect to the partial order in $\mathbf{R}^{d}$ ) solution of the Schröder equation

$$
1-G(F(x))=\rho(1-G(x)), \quad G(0)=0 .
$$

This is accomplished by linearization and reduction to one dimension. It is then shown that the lim sup of any subsequence of $(2 b)$ is also a solution of (3), which proves that the entire sequence converges.

Methodology. Define

$$
G_{n}(x)=v \cdot\left(F_{n}(x)-F_{n}\right) / v \cdot\left(1-F_{n}\right),
$$

and

$$
G(x)=\limsup _{n \rightarrow \infty} G_{n}(x) .
$$

Obviously $G(x)$ is convex, monotonic nondecreasing, and satisfies $G(0)=0$. 
$G(x)$ will now be shown to be the unique solution of (3) with these properties. First, $G_{n}(F(x))$ may be expressed as $\left(a_{n}+b_{n}-1\right) / b_{n}$ where $a_{n}=G_{n+1}(x)$ and $b_{n}=v \cdot\left(1-F_{n}\right) / v \cdot\left(1-F_{n+1}\right)$. By (1b), $b_{n} \rightarrow \rho^{-1}$ and passage to the limit shows that $G(x)$ satisfies (3).

Let $P(x)$ denote any appropriate solution to (3). For $0 \leqslant s \leqslant 1$ and $n$ a positive integer define

$$
A_{n}(s)=\rho^{-n}\left[1-P\left(1-s\left(1-F_{n}\right)\right)\right] .
$$

Since $F$ is convex, $F\left((1-s) 1+s F_{n}\right) \leqslant(1-s) 1+s F_{n+1}$, and since $P$ is nondecreasing,

$$
\begin{aligned}
1-P\left((1-s) 1+s F_{n+1}\right) & \leqslant 1-P\left(F\left((1-s)+s F_{n}\right)\right) \\
& =\rho\left(1-P\left((1-s) 1+s F_{n}\right)\right),
\end{aligned}
$$

this last equality by (3). Dividing by $\rho^{n+1}$ it follows that $A_{n+1}(s) \leqslant A_{n}(s)$ and therefore $A(s) \equiv \lim _{n \rightarrow \infty} A_{n}(s)$ exists. In particular, notice that $A(s)$ is concave, nondecreasing, with $A(1)=1$.

Next it is shown that $A(s) \equiv s$. To this end fix $0<s<1$ and let $\varepsilon>0$ be arbitrary but satisfying $s \rho(1+\varepsilon)<1$. From (1a) and (1b) for all sufficiently large $n$,

$$
(1-\varepsilon) \rho\left(1-F_{n}\right) \leqslant 1-F_{n+1} \leqslant(1+\varepsilon) \rho\left(1-F_{n}\right)
$$

whence

$$
\begin{aligned}
1-P\left(1-\rho s(1-\varepsilon)\left(1-F_{n}\right)\right) & \leqslant 1-P\left(1-s\left(1-F_{n+1}\right)\right) \\
& \leqslant 1-P\left(1-\rho s(1+\varepsilon)\left(1-F_{n}\right)\right) .
\end{aligned}
$$

Consequently,

$$
A_{n}(s \rho(1+\varepsilon)) \leqslant \rho A_{n+1}(s) \leqslant A_{n}(s \rho(1+\varepsilon))
$$

which implies that

$$
A(s \rho(1+\varepsilon)) \leqslant \rho A(s) \leqslant A(s \rho(1-\varepsilon)) .
$$

Letting $\varepsilon \downarrow 0$ it is seen that $A(s)$ satisfies

$$
A(\rho s)=\rho A(s)
$$

According to Kuczma (1964) equation (5) possesses a unique one parameter family of concave solutions given by $c s$ where $c$ is a constant. In this case $c$ is obviously equal to 1 , so summarizing,

$$
\lim _{n \rightarrow \infty} \rho^{-n}\left[1-P\left(1-s\left(1-F_{n}\right)\right)\right]=s
$$

(6) will now be used to force uniqueness. Fix $x \in[0,1]^{d}$ and suppose that 
$G(x) \neq 0$. Let $\left\{n^{\prime}\right\}$ denote a subsequence of the integers along which the lim sup is achieved in (4) for this particular choice of $x$. Thus

$$
\lim _{n^{\prime} \rightarrow \infty} v \cdot\left(1-F_{n^{\prime}}(x)\right) / v \cdot\left(1-F_{n^{\prime}}\right)=1-G(x) .
$$

Choose $\varepsilon>0$ arbitrary but satisfying $(1+\varepsilon)(1-G(x))<1$. Involing (1a) and (7), for all sufficiently large $n^{\prime}$,

$(8)(1-\varepsilon)(1-G(x))\left(1-F_{n^{\prime}}\right) \leqslant 1-F_{n^{\prime}}(x) \leqslant(1+\varepsilon)(1-G(x))\left(1-F_{n^{\prime}}\right)$

from which it obtains that

$$
\begin{aligned}
1-P(1-(1-\varepsilon) & \left.(1-G(x))\left(1-F_{n^{\prime}}\right)\right) \\
& \leqslant 1-P\left(F_{n^{\prime}}(x)\right) \leqslant 1-P\left(1-(1+\varepsilon)(1-G(x))\left(1-F_{n^{\prime}}\right)\right) .
\end{aligned}
$$

Divide these inequalities by $\rho^{n^{\prime}}$, use (6) to conclude

$$
(1-\varepsilon)(1-G(x)) \leqslant 1-P(x) \leqslant(1+\varepsilon)(1-G(x))
$$

and then let $\varepsilon \downarrow 0$ to deduce $P(x)=G(x)$. If $G(x)=0$ then proceed in an analogous fashion using the inequalities

$$
F_{n^{\prime}} \leqslant F_{n^{\prime}}(x) \leqslant F_{n^{\prime}}+\varepsilon\left(1-F_{n^{\prime}}\right)
$$

in place of $(8)$ to deduce that $P(x)=0$. This separate argument is necessitated because the coefficient of $1-F_{n^{\prime}}$ in the extreme right-hand inequality of (8) does not lie in $[0,1]$ and so (6) is not applicable. (Actually (6) may be extended to all $s>0$, since eventually $s\left(1-F_{n^{\prime}}\right)$ is less than 1.) Since $x$ is arbitrary it follows that $P(x)=G(x)$, proving the uniqueness assertion above.

Finally, to show that $\lim G_{n}(x)$ exists, let $\left\{n^{\prime}\right\}$ denote an arbitrary subsequence of the integers and let $H(x)=\lim \sup _{n^{\prime} \rightarrow \infty} G_{n^{\prime}}(x)$. Now $1-G_{n^{\prime}+1}(x)$ $=1-G_{n^{\prime}}(x) a_{n^{\prime}}, b_{n^{\prime}}$ where

$$
a_{n^{\prime}}=\frac{v \cdot\left(1-F_{n^{\prime}+1}(x)\right)}{v \cdot\left(1-F_{n^{\prime}}(x)\right)}, \quad b_{n^{\prime}}=\frac{v \cdot\left(1-F_{n^{\prime}}\right)}{v \cdot\left(1-F_{n^{\prime}+1}\right)},
$$

and $a_{n^{\prime}} b_{n^{\prime}} \rightarrow 1$ from (1b). Therefore one also has $H(x)$ $=\lim \sup _{n^{\prime} \rightarrow \infty} G_{n^{\prime}+1}(x)$. Imitate the steps in the first paragraph of this section to conclude that $H(x)$ satisfies (3) and then by uniqueness of solutions, $H(x) \equiv G(x)$. This obviously implies that $\lim _{n \rightarrow \infty} G_{n}(x)$ exists.

Concluding remarks. In a previous paper (1975a) the author proved that there is a unique p.g.f. solution to equation (3) which therefore characterized the Yaglom limit. The proof required differentiation of (3) to show that all p.g.f. solutions have the regularly varying property

$$
1-G(1-t u)=t L(t), \quad t \rightarrow 0,
$$

where $L(t)$ is slowly varying at 0 . (Such an approach is not valid here since the 
function $G(x)$ defined by (4) is not a priori differentiable.) The essence of uniqueness was in the property (9) used in the following form:If $s_{n}$ and $t_{n}$ tend to zero in such a way that $s_{n} / t_{n} \rightarrow a$ and $0<a<\infty$, then

$$
\lim _{n \rightarrow \infty} \frac{1-G\left(1-s_{n} u\right)}{1-G\left(1-t_{n} u\right)}=a .
$$

Now, letting $s_{n}=s\left(1-F_{n}\right)$ and $t_{n}=t\left(1-F_{n}\right)$, where $s / t=a$, then (10) also results but this time as a consequence of (6). Moreover by combining (6) and (9) it follows that if $t_{n}^{\prime}$ and $t_{n}$ tend to zero in such a way that $t_{n}^{\prime} \rho^{n}$ $\sim t_{n} v \cdot\left(1-F_{n}\right)$ then $1-G\left(1-t_{n}^{\prime} u\right) \sim t_{n}$. Such a result is related to the more general problem of inversion of asymptotic relationships involving slowly varying functions (see Bojanic and Seneta (1971), §3).

The technique developed here depends only upon the behaviour of the iterates of convex functions at a fixed point. It is therefore highly probable that it can be extended and applied to the more general problem of the existence and uniqueness of solutions to the multidimensional Schröder equation. A similar approach leading to a limit relationship analogous to (6) has been used by the author (1977) in the investigation of invariant measures on Galton-Watson processes and in that setting it is Abel's equation which is of relevance.

Thanks are due to Professor Anatole Joffe for a number of stimulating conversations and, in particular, for first expressing interest in a nonprobabilistic proof.

\section{REFERENCES}

1. R. Bojanic and E. Seneta (1971), Slowly varying functions and asymptotic relations, J. Math. Anal. Appl. 34, 302-315.

2. C. R. Heathcote, E. Seneta and Vere-Jones (1967), A refinement of two theorems in the theory of branching processes, Theor. Probability Appl. 12, 297-301.

3. F. M. Hoppe (1975a), Stationary measures for multitype branching processes, J. Appl. Probability 12, 219-227.

4. (1975b), Functional equations with applications to multitype Galton-Watson branching processes, Doctoral Dissertation, Princeton Univ., Princeton, N.J.

5. (1977), Representations of invariant measures on multitype Galton-Watson processes, Ann. Probability 5, 291-297.

6. ___ (1977), The critical Bienaymé-Galton-Watson process, Stochastic Processes Appl. 5, $57-66$.

7. A. Joffe and F. Spitzer (1967), On multitype branching processes with $\rho<1$, J. Math. Anal. Appl. 19, 409-430.

8. M. Kuczma (1964), Note on Schröder's functional equation, J. Austral. Math. Soc. 4, 149-151.

9. E. Seneta (1969), Functional equations and the Galton-Watson process, Advances in Appl. Probability 1, 1-42.

Department of Mathematics, University of Alberta, Edmonton, Alberta T6G 2gl, Canada 